Hydroécol. Appl. (2000) Tome 12 Vol, 1-2, pp. 159-182

\title{
Comportement des métaux traces dans le bassin versant de l'Orne. Variabilité saisonnière et bilan des transferts (1994-1995)
}

\section{Trace metals chemistry in the Orne river, France. Seasonal variability and chemical fluxes (1994-1995)}

\author{
A. Nasseh (1-2), H. Texier ${ }^{(2)}$ \\ (1) Ecole Normale Supérieure, Département de Physique, B.P. 209, Martil, Tétouan, Maroc. \\ (2) Centre de CNRS, 24 rue du tilleul, Université de Caen, 14032 Caen Cedex.
}

Résumé. - De septembre 1993 à décembre 1995, une étude relativement complète portant sur les variations saisonnières des concentrations et des flux des contaminants inorganiques en traces a été menée sur le bassin de l'Orne. Ce bassin, de taille moyenne $\left(2900 \mathrm{~km}^{2}\right)$, constitue un atelier remarquable de par son régime hydroclimatique et sa géomorphologie contrastés.

Les prélèvements ont été effectués à la station exutoire N11 du bassin ainsi qu' en dix stations du réseau hydrographique, correspondant notamment aux discontinuités lithologiques et hydrologiques. Les analyses présentées ont essentiellement porté sur les teneurs en métaux traces $(\mathrm{Cd}, \mathrm{Cr}, \mathrm{Cu}, \mathrm{Ni}, \mathrm{Pb}, \mathrm{Zn}$ ) des eaux et des particules de cette station.

Les concentrations particulaires des métaux $\mathrm{Cu}, \mathrm{Cr}$ et Ni reflètent globalement les teneurs observées dans les substrats naturels. Par contre, le $\mathrm{Cd}$, le $\mathrm{Pb}$ et le $\mathrm{Zn}$ montrent des teneurs importantes ( 8 à 17 fois la moyenne de fleuves mondiaux considérés peu contaminés). Cette contamination est confirmée par les valeurs du facteur d'enrichissement calculées pour les sédiments de fond. Les coefficients de distribution moyens $\left(K_{4}\right)$ sont comparables à ceux observés dans d'autres fleuves mondiaux $\left(\log \mathrm{K}_{n}\right): \mathrm{Cd}=5,0 ; \mathrm{Cu}=4,9 ; \mathrm{Cr}=4,8 ; \mathrm{Pb}=5,4 ; \mathrm{Zn}=5,0 ; \mathrm{Ni}=5,1$. Ils montrent que les concentrations sont essentiellement liées à la matière particulaire.

L'étude a montré que les stratégies de surveillance et de calcul des flux polluants doivent être adaptées aussi bien au cycle hydrologique qu'aux variations des concentrations des MES.

Mots-clés. - rivières, métaux en traces, coefficient de distribution, flux.

Abstract. - Data on seasonal concentration variations and annual fluxes for trace metals are presented for the Orne basin (Calvados, France): area $2900 \mathrm{~km}^{2}$. The work is based on the monthly monitoring of 11 sites along the main river course; particularly at 
points of lithological and hydrographic discontinuity; the analysis focuses on the concentrations in dissolved and particulate trace elements $(\mathrm{Cd}, \mathrm{Cr}, \mathrm{Cu}, \mathrm{Ni}, \mathrm{Pb}, \mathrm{Zn})$.

The concentrations of most trace elements studied are similar to those in the bedrock. On the other hand, cadmium contamination appears high ( 8 times the mean for the world's unpolluted rivers); this is confirmed by the calculated enrichment factor values for bottom sediment.

The trace metal concentrations show complex spatial variations. During floods, these concentrations are diluted. Median partitioning coefficients $(K d)$ are similar to those found in other world rivers $(\log K d)$ : $\mathrm{Cd}=0.5 ; \mathrm{Cu}=4.9 ; \mathrm{Cr}=4.8 ; \mathrm{Pb}=5.4 ; \mathrm{Zn}=5.0 ; \mathrm{Ni}=5.1$. They show that concentrations are essentially linked to the particulate matter.

The study showed that strategies for monitoring and calculating pollutant fluxes must be well adapted not only to the hydrological cycle but also to variations in SM concentrations.

Key-words. - rivers, trace metals, distribution coefficients, fluxes.

\section{INTRODUCTION}

Les fleuves sont le véhicule d'une grande partie des substances issues des activités industrielles, agricoles et domestiques. La connaissance des apports en contaminants chimiques par les rivières à leurs estuaires est fondamentale aussi bien pour leur gestion et leur aménagement que pour évaluer les apports à l'océan. Ils ont fait l'objet à ce titre de nombreux travaux (Abdel-Moati, 1990; Shankar et Manjunatha, 1994 ; Zhang, 1995; Guieu et al., 1996; Idlafkih et al., 1997; Meybeck, 1998; Lin et Chen, 1998; Mueller et Furrer, 1998).

L'étude des mécanismes qui règlent ces flux polluants nécessite, entre autres, une meilleure connaissance de la dynamique des transferts en fonction des différents facteurs influants (climat, régime hydrologique, lithologie, relief, apports anthropiques...) (Taleb, 1997).

Nous nous sommes intéressés dans ce travail au comportement des contaminants en traces inorganiques dans l'Orne ( $\mathrm{Cd}, \mathrm{Cu}, \mathrm{Cr}, \mathrm{Pb}, \mathrm{Zn}$ et $\mathrm{Ni}$ ). Ceux-ci se trouvent dans les eaux naturelles sous différentes formes chimiques (ions libres, complexes, formes particulaires, ...) et constituent des marqueurs importants de la plupart des pollutions anthropiques. Nous exposons dans ce travail les résultats d'une étude menée dans un petit bassin versant agricole situé en région tempérée. Ils concernent les niveaux de ces contaminants en fonction de l'espace, du temps et du débit de la rivière et permettent d'établir des bilans.

\section{SITE D'ÉTUDE}

Le bassin versant de l'Orne, centré en Basse-Normandie, est le plus vaste de cette région. Sa superficie totale est de près $2900 \mathrm{~km}^{2}$. Le fleuve Orne, d'une longueur de $175 \mathrm{~km}$, prend sa source à une altitude de $218 \mathrm{~m}$, au sud-sud-est de Sées et se 


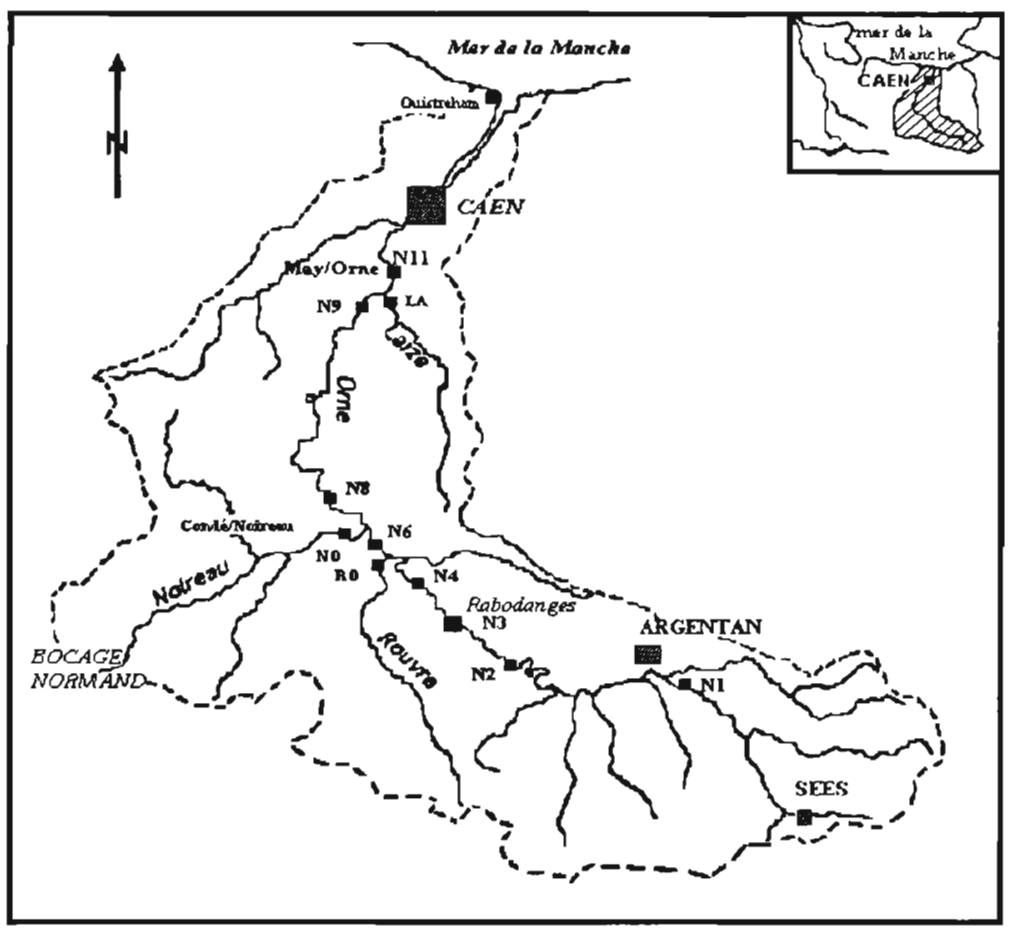

Fig. 1. - Bassin de l'Orne et situation des stations de prélèvements

Fig. 1. - Orne Basin and location of sampling sites.

jette dans la mer de la Manche à Ouistreham (fig. 1).

Le bassin de l'Orne a une vocation essentiellement agricole. Les campagnes d'Argentan, de Caen et de la Laize $(40 \%$ de la superficie totale du bassin) sont les principales régions agricoles du bassin (cultures industrielles et céréalières essentiellement). Dans le Bocage Normand et les fonds de vallées, on trouve principalement de l'élevage. L'activité industrielle est concentrée principalement dans l'agglomération de Caen et sa grande ceinture d'unités urbai- nes (sidérurgie portuaire, industries automobile et agroalimentaires).

La proximité de la mer et sa position relativement basse en latitude font que le bassin versant de l'Orne bénéficie d'un régime climatique typiquement océanique dominé par des pluies fines réparties sur toute l'année. Les précipitations abondantes sont très inégalement réparties et montrent une opposition remarquable entre les campagnes carbonatées de Caen et d'Argentan (lame d'eau inférieure à $650 \mathrm{~mm}$ en moyenne) et les reliefs silico-alumi- 
neux du Bocage (plus de $900 \mathrm{~mm}$ en moyenne) (Nasseh et al., 1996).

Le débit de la rivière subit des fluctuations très importantes, avec des valeurs extrêmes de moins de $2 \mathrm{~m} / \mathrm{s}$ en étiage à parfois plus de $200 \mathrm{~m}^{3} / \mathrm{s}$ en crue.

Le réseau hydrographique de l'Orne reflète la diversité lithologique du bassin. Mais ce sont principalement les rivières de rive gauche, aux longueurs de drains et aux bassins les plus conséquents qui alimentent le cours de l'Orne (Nasseh, 1997).

\section{MATÉRIEL ET MÉTHODES}

Les données des métaux en trace utilisées dans cette étude ont été recueilles durant deux cycles hydrologi- ques complets (1994 et 1995) en onze stations réparties sur le bassin versant, entre Argentan et Caen (fig. 1). Les positions de ces stations ont été choisies en fonction des caractéristiques lithologiques et hydrologiques du bassin. Trois stations ont été fixées à la confluence des principaux affluents de l'Orne afin d'en déterminer l'apport et l'influence: station RO sur la Rouvre, station NO sur le Noireau et station LA sur la Laize. Les prélèvements sont effectués mensuellement en ces stations (264 échantillons). La station N11 de May-sur-Orne, point le plus aval de l'Orne non soumis aux influences de la marée dynamique, a été le siège d'un suivi plus serré : prélèvements généralement hebdomadaires en hiver et quotidiens pendant les crues (fig. 2). Ceci nous a permis de dispo-

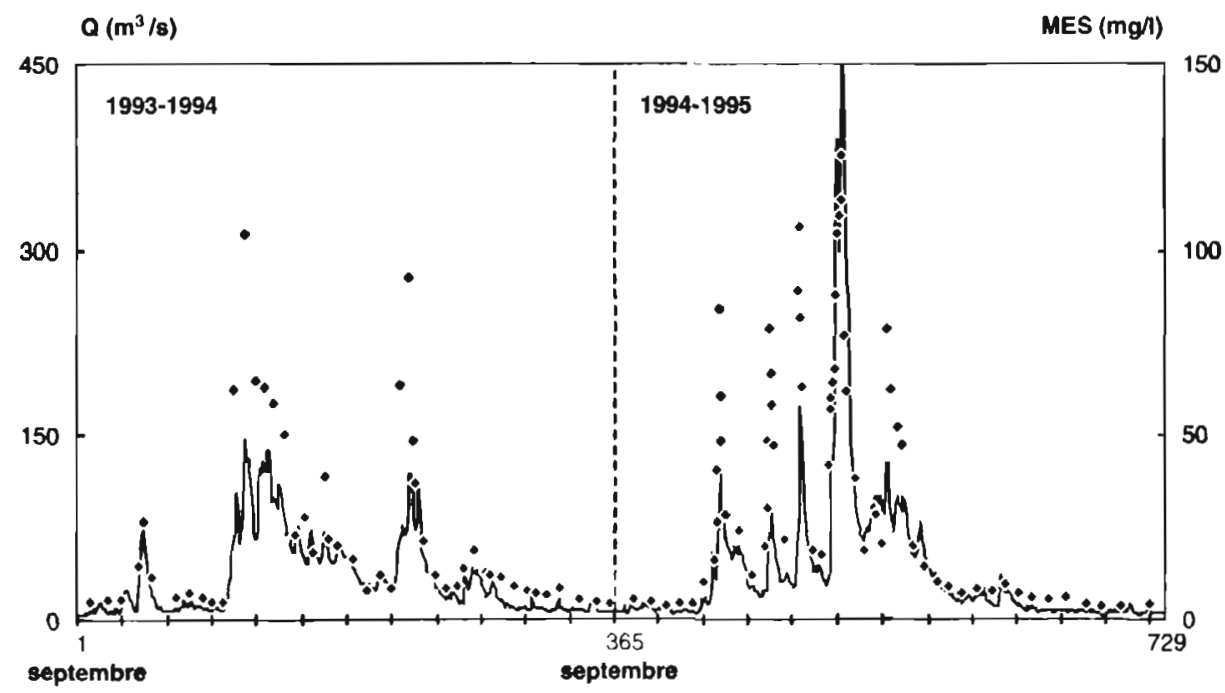

Fig. 2. - Variation des débits et des concentrations en MES les jours de prélévements à May/Orne (1993-95).

Fig. 2. - Evolution of discharge and suspended matter concentrations in the Orne (1993-95). 
ser en cette station de 102 échantilIons sur deux ans.

Les prélèvements instantanés d'eau ont été réalisés au milieu du cours d'eau. Des mesures in situ de différents paramètres physico-chimiques des eaux sont effectuées à chaque campagne de prélèvement $\left(t^{\circ} \mathrm{C}, \mathrm{pH}\right.$, conductivité). Plusieurs litres d'eau sont recueilles à chaque station dans des flacons qui ont subi tous les lavages nécessaires.

Les méthodes de lavage du matériel et d'analyses sont décrites dans Ouddane (1990).

Les flacons, les appareils de filtration ainsi que le matériel de laboratoire utilisés sont lavés au détergent puis lavés abondamment à l'eau distillée. Les flacons sont ensuite remplis d'un mélange acide pendant une dizaine de jours puis abondamment rincés à l'eau Milli-Q. Le reste du matériel est stocké dans du $\mathrm{HNO}_{3}(1: 3)$ puis dans du $\mathrm{HCl}(1: 3)$ (acid Merck Suprapur) pendant deux semaines, puis lavé abondamment à l'eau Milli$Q$ avant utilisation.

L'eau est filtrée dans les heures qui suivent les prélèvements sur des filtres de porosité $0,45 \mu \mathrm{m}$ qui ont été plongés dans une solution acidifiée pendant 48 heures et lavés abondamment à l'eau Milli-Q. Les analyses sont ensuite effectuées sur le filtrat acidifié (acidification à $\mathrm{pH}=2$ avec $\mathrm{HCl}$ " Merck Suprapur») et sur la matière en suspension.

Les métaux en traces dissous ( $C d$, $\mathrm{Cr}, \mathrm{Cu}, \mathrm{Pb}, \mathrm{Ni}$ et $\mathrm{Zn}$ ) ont été analysés essentiellement par redissolution anodique en mode impulsionnel différentiel.

Les réactifs utilisés dans la minéralisation des particules pour le dosage des métaux sont de qualités "Suprapur". Les éléments métalliques sont analysés à l'aide d'un spectromètre d'absorption atomique Perkin Elmer 2380 équipé d'un four HGA 500 avec passeur automatique AS 40 .

Les mesures ont aussi concerné divers éléments majeurs et mineurs : $\mathrm{Mg}, \mathrm{K}, \mathrm{Ca}, \mathrm{Na}, \mathrm{SiO}_{2}, \mathrm{HCO}_{3}, \mathrm{SO}_{4}{ }^{2 \cdot}, \mathrm{Cl}$, $\mathrm{NO}_{3}, \mathrm{PO}_{4}^{3}, \mathrm{NH}_{4}, \mathrm{Al}, \mathrm{Fe}$ et Ti. Seul le résultat global annuel sera utilisé pour comparer les flux de métaux traces.

Les bilans annuels d'exportation ont été établis pour les flux dissous à partir de la formule :

$$
F=\Sigma F_{i m}
$$

$F_{i m}$, est le flux instantané calculé à partir de la relation $F=f\left(Q_{1}\right)$ établie par modélisation statistique à partir des mesures instantanées $\left(C_{i}\right.$ et $\left.Q_{i}\right)$ des concentrations et du débit (fig. 7);

et pour les flux particulaires à partir de la formule :

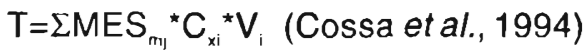
où $\mathrm{MES}_{m j}$, est la teneur en MES moyenne journalière calculée à partir de la relation $M E S_{1}=20,7^{*} \operatorname{Ln}\left(Q_{1}\right)-40,6$ établie par modélisation statistique $\left(R^{2}=0,77\right)$ à partir des mesures instantanées (MES et $Q_{1}$ ) des MES et du débit ; $C_{x i}$ teneur de l'élément $x$ dans les MES de l'échantillon instantané; $V_{i}$ : volume d'eau écoulé sur l'intervalle de temps pris autour de la date 
de mesure symétriquement entre deux prélèvements successifs (Nasseh et al., 1999).

\section{RÉSULTATS ET DISCUSSION}

\subsection{Variabilité des débits liquide et solide}

Le régime hydrologique représente la principale cause de variation des transports en solution. Les crues constituent des événements essentiels dans ce cycle pour comprendre l'origine et la dynamique de transfert des éléments qui sont exportés par les fleuves (Edwards, 1973; Meade et Parker, 1984). Les matières en suspension sont considérées quant à elles comme un vecteur essentiel de la pollution en métaux, micropolluants organiques et matières organiques dans les milieux aquatiques.

Durant la période d'étude, les conditions climatiques se sont fortement écartées des conditions moyennes. Le débit moyen annuel mesuré à May-sur-Orne des deux cycles étudiés, respectivement $33 \mathrm{~m}^{3} / \mathrm{s}$ et $39 \mathrm{~m}^{3} / \mathrm{s}$, est élevé par rapport au débit moyen interannuel $\left(20,5 \mathrm{~m}^{3} / \mathrm{s}\right)$ calculé sur les neufs derniers cycles hydrologiques (1985-1993).

L'Orne a connu au cours de cette période plusieurs crues dont une crue centennale (janvier-février 1995) (fig. 2). La durée des crues ainsi que les volumes d'eau écoulés montrent que $60 \%$ du volume d'eau total se sont écoulés en $24 \%$ du cycle en 1993-1994, et environ $70 \%$ se sont écoulés en $25 \%$ du temps en 19941995 (Tab. I). La crue de février 1995 a écoulé $676106 \mathrm{~m}^{3}$ soit $54,5 \%$ du volume total du cycle pendant $9 \%$ du temps. Elle a duré plus de vingt jours et atteint $452 \mathrm{~m}^{3} / \mathrm{s}$. La nature principalement imperméable des terrains briovériens et les fortes pentes qui les marquent, renforcées par les contraintes anthropiques qui lui sont imposées, font du bassin un milieu propice aux phénomènes de crue et d'inondation. Les montées de crue sont généralement rapides.

Le régime des MES est très variable et est très dépendant du débit liquide (fig. 2). La charge moyenne en suspension à la sortie du bassin versant s'élève sur les deux cycles étudiés à $52,5 \mathrm{mg} / \mathrm{l}$. En période d'étiage, les concentrations des MES varient de quelques milligrammes par litre à

Tableau I. - Contributions des crues au volume total écoulé au cours des cycles hydrologiques.

Table I. - Floods contributions to the total water volume during the hydrological cycles.

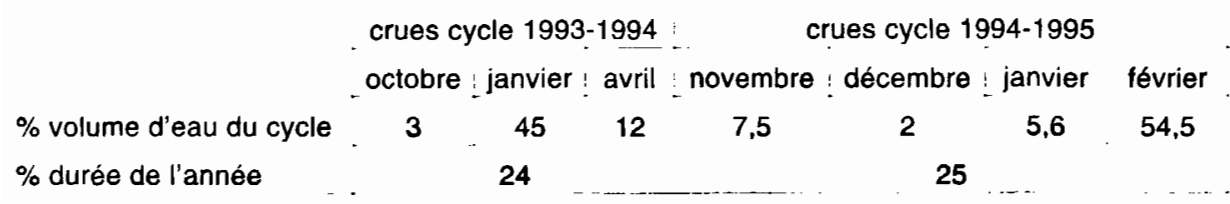


$20 \mathrm{mg} / \mathrm{l}$. L'analyse microgranulométrique montre que les tailles des populations des particules sont centrées sur 9-11 $\mu \mathrm{m}$ (Nasseh, 1997; Nasseh et al., 1999). Le développement planctonique est la source principale de particules en suspension en cette période.
En période des hautes eaux le lessivage des sols du bassin ainsi que la remise en suspension des sédiments meubles de fond du fleuve entraînent une augmentation importante des concentrations en MES qui atteignent $125 \mathrm{mg} / \mathrm{l}$ en crue. Les spectres granulométriques montrent un étalement

Tableau II. - Comparaison des concentrations moyennes en ng/l de métaux traces en solution dans les eaux de l'Orne (May/Orne) et d'autres fleuves français et mondiaux.

Table Il. - Comparison of the heavy metal concentrations (in $\mathrm{ng} / \mathrm{l}$ ) in Orne water with some other rivers in France and worldwide.

\begin{tabular}{|c|c|c|c|c|c|c|c|}
\hline & $\mathrm{Cd}$ & $\mathrm{Cu}$ & $\mathrm{Cr}$ & $\mathrm{Pb}$ & $\mathrm{Zn}$ & $\mathrm{Ni}$ & références \\
\hline Orne & 29 & 780 & 640 & 215 & 2100 & 280 & cette étude $(1993 / 1995)$ \\
\hline Seine & 30 & 1890 & $=$ & 458 & 11540 & - & Idlafkih et al., 1995 \\
\hline Rhône & 47.9 & 1880 & - & 92 & 1050 & & Elbaz-Poulichet, 1988 \\
\hline Rhin & 30.1 & 4500 & - & 1020 & 449001 & - & $\begin{array}{l}\text { V. der Weijden et } \\
\text { Middelburg, } 1989\end{array}$ \\
\hline Léna & 6 & 880 & - & 52 & 78.5 & 258 & Guieu ef al., 1996 \\
\hline Huanghe & 2.2 & 1386 & - & 29 & 229 & 405 & Zhang et al., 1994 \\
\hline Changjiang & 3.4 & 1650 & - & 54 & 58.9 & 147 & in Zhang, 1995 \\
\hline moyenne mondiale & 20 & 1500 & $1000 i$ & 100 & 30000 & 500 & Martin et Whitfield, 1983 \\
\hline
\end{tabular}

Tableau III. - Concentrations moyennes en $\mu \mathrm{g} / \mathrm{g}$ des éléments traces particulaires dans les MES de l'Orne et de certaines rivières françaises.

Table III. - Heavy metal concentrations $(\mu \mathrm{g} / \mathrm{g})$ in suspended sediments in Orne water and in some other French rivers.

\begin{tabular}{|c|c|c|c|c|c|c|c|}
\hline & $\mathrm{Cd}$ & $\mathrm{Cu}$ & $\mathrm{Cr}$ & _ $\mathrm{Pb}$ & $\mathrm{Zn}$ & $\mathrm{Ni}$ & références \\
\hline Orne & 2.8 & 80 & 65 & 72 & -235 & 52 & cette étude \\
\hline Seine & 4.95 & 174 & - & 184 & 611 & - & Idlafkih et al., 1995 \\
\hline Rhône & 1.7 & 100 & - & 97 & 279 & - & Elbaz-Poulichet, 1988 \\
\hline Rhin & 32 & 260 & - & 425 & 1600 & - & $\begin{array}{l}\text { V. der Weijden et } \\
\text { Middelburg., } 1989\end{array}$ \\
\hline Garonne & - & 51 & 255 & - & 874 & 33 & Meybeck, 1984 \\
\hline Huanghe & 0.18 & 26.7 & 76.9 & 16.4 & 69.8 & 40.3 & Zhang et al., 1995 \\
\hline Léna & - & 28 & - & 23 & 143 & $=$ & in Cossa et al., 1994 \\
\hline moyenne mondiale & 1 & 100 & 100 & 100 & 250 & 90 & Martin et Whitfield, 1983 \\
\hline
\end{tabular}


général vers les grandes tailles. II correspond à l'arrivée de petits agrégats de sols associés aux processus d'érosion des sols. Les tailles des populations sont comprises entre 3 et 33-35 $\mu \mathrm{m}$.

\subsection{Niveaux de concentration}

Les concentrations moyennes pondérées par le débit des éléments traces dissous et particulaires mesurées à l'exutoire du bassin, à May/Orne, sont comparées dans les tableaux II et III à certaines rivières françaises ainsi qu'aux teneurs moyennes de rivières mondiales considérées peu affectées par les apports anthropiques (Léna, Huanghe et Changjiang ; Cossa et al., 1994).

Mis à part le Cu et le Ni qui présentent des teneurs plus faibles ou comparables à celles des rivières peu affectées par les apports anthropiques, les concentrations en solution observées dans l'Orne à May-surOrne pour les autres éléments sont plus élevées. Elles représentent huit fois les concentrations dans les fleu- ves de référence pour le cadmium, cinq fois pour le plomb et dix sept fois pour le zinc. Néanmoins, ces valeurs restent plus faibles (sauf pour $\mathrm{Cd}$ et $\mathrm{Pb}$ ) que la composition moyenne des rivières donnée par Martin et Whitfield (1983).

Les teneurs dans les MES montrent des concentrations en $\mathrm{Cd}, \mathrm{Cu}$, $\mathrm{Pb}$ et $\mathrm{Zn}$ plus élevées que dans les fleuves de référence. Sauf pour le Cd, ces valeurs restent plus faibles que la composition moyenne des rivières donnée par Martin et Whitfield (1983). Elles sont dans l'ensemble comparables aux teneurs observées dans les substrats $(\mathrm{Cu}, \mathrm{Cr}, \mathrm{Zn}, \mathrm{Ni})$ (tab. IV). La pollution en cadmium est confirmée par le facteur d'enrichissement (FE) calculé à partir du rapport des teneurs dans les sédiments de fond et dans les substrats, normalisées à l'Al (FE > 1.8; Nasseh et al., 1999).

Ces concentrations dissoutes et particulaires ne montrent pas, sauf pour le cadmium, de pollution marquée des eaux en métaux lourds. Ceci montre que la plupart de ces élé-

Tableau IV. - Teneurs moyennes en métaux traces $(\mu \mathrm{g} / \mathrm{g}$ ) dans les principaux substrats du bassin de l'Orne (Nasseh, 1997).

Table IV. - Trace metals concentrations $(\mu \mathrm{g} / \mathrm{g})$ in Orne basin substratum.

\begin{tabular}{|c|c|c|c|c|c|c|}
\hline & Cd & $\mathrm{Cu}$ & $\mathrm{Cr}$ & $\mathrm{Pb}$ & $\mathrm{Zn}$ & $\mathrm{Ni}$ \\
\hline Schistes et grès & 0,54 & 93,5 & 107,0 & 13,2 & 150 & 62,6 \\
\hline schistes & 0.76 & 52,1 & 121,4 & 3,5 & 128 & 47,1 \\
\hline grès & 0,19 & 46,5 & 112,6 & 1,05 & 124 & 56,6 \\
\hline quartzites & 0,24 & 15,0 & 83.1 & 24,0 & 210 & 31,7 \\
\hline calcaires & 0,22 & 39,0 & 80.9 & 5,2 & 82 & 30,5 \\
\hline
\end{tabular}


Tableau V. - Taux d'exportation spécifique dans l'Orne, la Léna et la Seine.

Table V. - Specific fluxes in the Orne, Lena and Seine rivers.

\begin{tabular}{lccccccl} 
& \multicolumn{8}{c}{ FORMES DISSOUTES } & $\left(\mathrm{kg} \cdot \mathrm{an}^{-1} \cdot \mathrm{km}^{-2}\right)$ & \\
& $\mathrm{Cd}$ & $\mathrm{Cu}$ & $\mathrm{Cr}$ & $\mathrm{Pb}$ & $\mathrm{Zn}$ & $\mathrm{Ni}$ & références \\
Orne & 0,007 & 0,12 & 0,11 & 0,093 & 0,74 & 0,097 & cette étude \\
Léna & 0,001 & 0,10 & - & 0,004 & 0,07 & - & in Cossa et al., 1994 \\
Seine & 0,004 & 0,13 & - & 0,070 & $1,20 \ldots$ & - & Cossa et al., 1994 \\
\hline
\end{tabular}

ments proviennent essentiellement de l'érosion du bassin de drainage. L'essentiel des sources de pollutions métalliques présentent dans le bassin versant de l'Orne, est situé au niveau de l'agglomération caennaise à l'aval de la station exutoire (N11). La pollution en Cd peut être due à l'utilisation massive en agriculture de fertilisants phosphatés (Elbaz-Poulichet et al., 1987).

Les flux dissous spécifique $\left(\mathrm{kg} \cdot \mathrm{an}^{-1} \cdot \mathrm{km}^{2}\right)$ montrent des valeurs importantes pour le cadmium et le plomb qui sont légèrement supérieures à celles de la Seine (tab. V).

\subsection{Variabilité spatiale et temporelle des concentrations}

Le tableau VI donne les moyennes arithmétiques des principaux sousbassins et à l'exutoire du bassin, ainsi que les statistiques générales sur l'ensemble des stations.

Les concentrations dissoutes montrent des variations relativement

Tableau VI. - Concentrations moyennes en teneurs dissoutes et particulaires des principaux sousbassins, et plages de variation sur l'ensemble du bassin de l'Orne (1993-1995).

Table VI. - Upstream-downstream evolution of the mean dissolved and particulate concentrations, and statistics on all the stations.

\begin{tabular}{|c|c|c|c|c|c|c|c|c|c|c|c|c|}
\hline \multirow[b]{2}{*}{ Stations } & \multicolumn{5}{|c|}{ teneurs dissoutes (ng/l) } & \multicolumn{7}{|c|}{ teneurs particulaires $(\mathrm{mg} / \mathrm{g}$ ) } \\
\hline & $\mathrm{Cd}$ & $\mathrm{Cu}$ & $\mathrm{Cr}$ & $\mathrm{Pb}$ & $\mathrm{Zn}$ & $\mathrm{Ni}$ & $\mathrm{Cd}$ & $\mathrm{Cu}$ & $\mathrm{Cr}$ & $\mathrm{Pb}$ & $\mathrm{Zn}$ & $\mathrm{Ni}$ \\
\hline Orne-amont & 18 & 649 & 543 & 202 & 1847 & 231 & 1.71 & 75 & 62.5 & 53.4 & 187 & 51.3 \\
\hline Rouvre (RO) & 21 & 673 & 528 & 191 & 1688 & 239 & 1.69 & 67 & 56.1 & 48.9 & 168 & 62.5 \\
\hline Noireau (NO) & 16 & 703 & 488 & 221 & 1923 & 216 & 1.64 & 80 & 64.2 & 57.4 & 198 & 52.2 \\
\hline Laize (LA) & 22 & 659 & 491 & 198 & 1776 & 219 & 1.80 & 70 & 59.3 & 51.8 & 179 & 49.1 \\
\hline May/Ome (N11) & 29 & 780 & 640 & 215 & 2100 & 280 & 2.80 & 80 & 65 & 72 & 235 & 52 \\
\hline moyenne & 20 & 670 & 531 & 206 & 1840 & 230 & 1.87 & 74 & 61.0 & 54 & 185 & 54 \\
\hline Ecart type & 6 & 215 & 193 & 59 & 368 & 65 & 0.24 & 5 & 4.3 & 5 & 11.5 & 4.6 \\
\hline CV (\%) & 30 & 32 & 36 & 29 & 20 & 28 & 13 & 7 & 7 & 9 & 6 & 9 \\
\hline
\end{tabular}



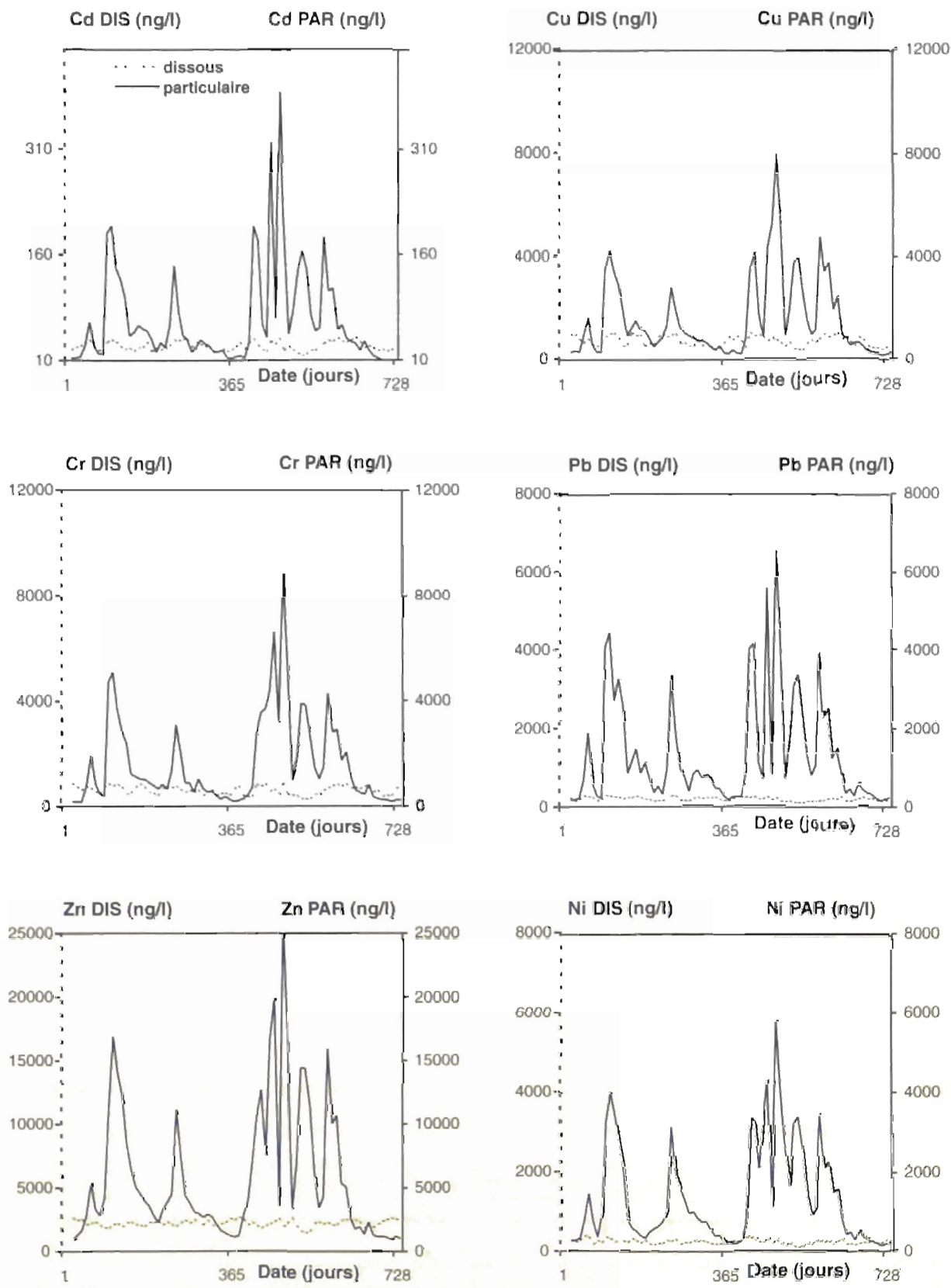

Fig. 3. - Variations temporelles des contaminants inorganiques dans les eaux de I' Orne (PAR: concentration particulaire; DIS: concentration dissoute).

Fig. 3. - Temporal variations in inorganic contaminants in Orne water (PAR: Particulate concentrations; DIS: Dissolved concentrations). 
importantes sur l'ensemble des mesures (valeurs du coefficient de variation CV comprises entre 20 et $36 \%$ ).

Les faibles variations amont-aval des concentrations particulaires sont significatives d'une relative homogénéité de la charge solide transportée par les eaux (Nasseh et al., 1999). Elles montrent en particulier que le $\mathrm{Cr}$ et le $\mathrm{Zn}$ proviennent essentiellement de l'amont et du Noireau, le $\mathrm{Cu}$ du Noireau et le Ni de la Rouvre.

Les formes dissoutes semblent plus fluctuantes que les formes particulaires (fig. 3). Elles sont plus affectées par l'effet de la dilution due entre autres à l'augmentation du débit vers l'aval. Exprimées en ng/l, les formes particulaires sont toujours dominantes par rapport aux formes dissoutes. La faible désorption de la phase particulaire vers la phase dissoute peut trouver son explication dans la faible proportion de métal échangeable par rapport au métal associé aux particules.

\subsection{Comportement lié au débit à May-sur-Orne}

Les concentrations des métaux en trace dissous en fonction du débit présentent des nuages de points très dispersés et varient dans des gammes très étendues (fig. 4). On observe lors des hautes eaux des variations du même ordre que celles observées en périodes d'étiages. Les teneurs ne dépendent pas uniquement du débit, elles sont également régulées par d'autres processus tels que la partition entre les phases solides et dissoutes.

Les variations des teneurs particulaires avec le débit ne montrent pas, elles non plus, de tendances particulières (fig. 5). Les teneurs diminuent fortement lors des grands débits du fait principalement des processus de dilution. Cette diminution peut être aussi due au mélange des sédiments pauvres en métaux érodés du bassin versant ou à l'effet des sédiments en suspension plus grossiers qui sont plus abondants en période de crue (Nasseh, 1997).

Les variations des concentrations des métaux particulaires avec les MES montrent des nuages de points relativement dispersés (fig. 6). On observe néanmoins une tendance à l'augmentation des teneurs avec les MES. Cependant, lorsque les teneurs en MES deviennent importantes lors des grandes crues, elles deviennent relativement moins riches en ces éléments. Ce comportement particulier est à mettre en relation avec la taille des particules transportées. Les faibles charges en MES transportées lorsque le débit est faible, sont constituées principalement de matériaux fins (argiles et limons) riches en métaux (Nasseh, 1997). Par contre, lorsque les débits sont élevés, les matériaux transportés par le cours d'eau sont plus lourds et plus grossiers (sables et graviers) et les teneurs en métaux sont plus faibles. Les faibles teneurs observées en général, pour la plupart des métaux, en période d'étiage, peuvent être imputées à la modification de la nature des 

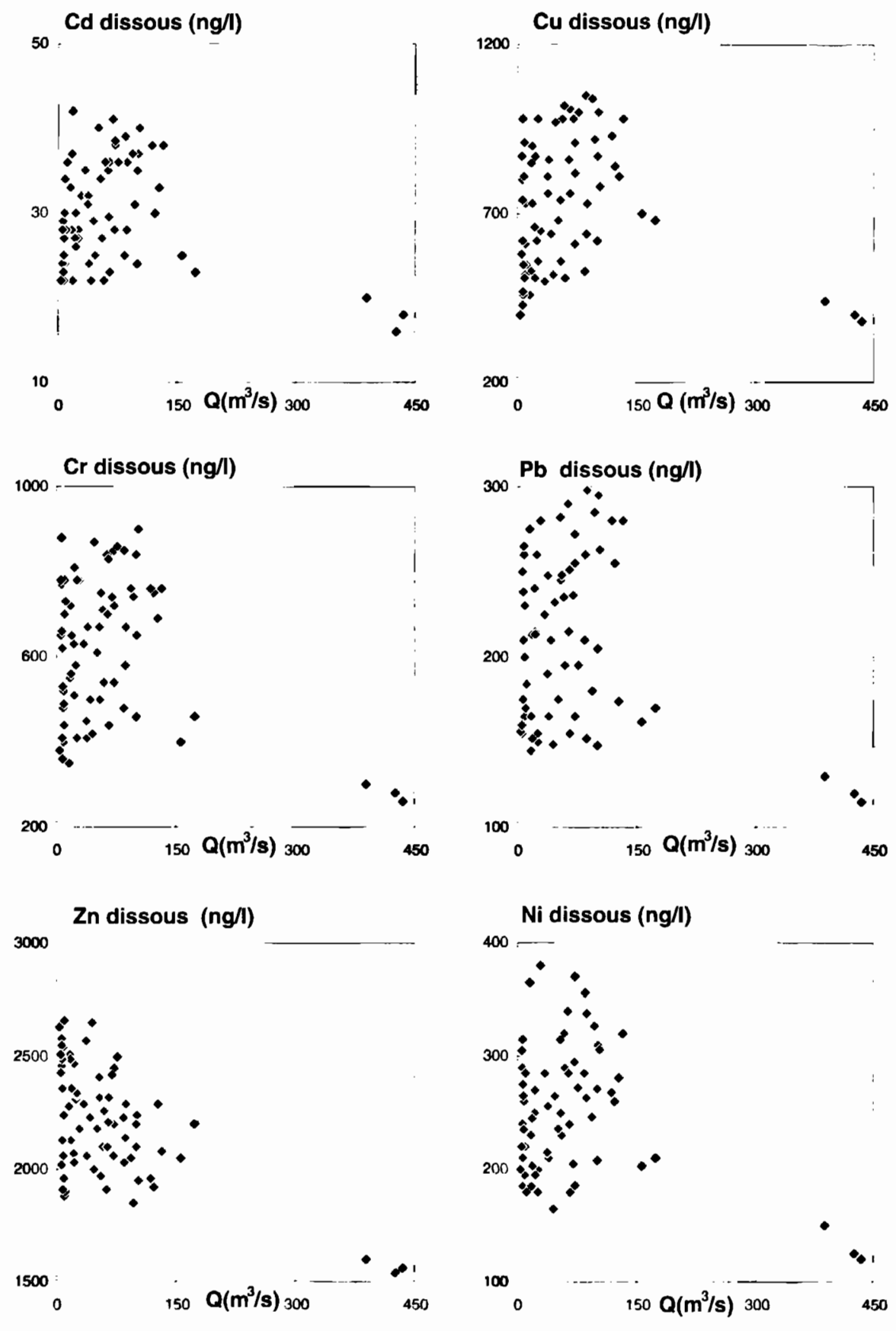

Fig. 4. - Variation des teneurs en métaux traces dissous dans les eaux de l'Orne (1993-1995). Fig. 4. - Variation in dissolved trace metal content in the Orne (1993-1995). 

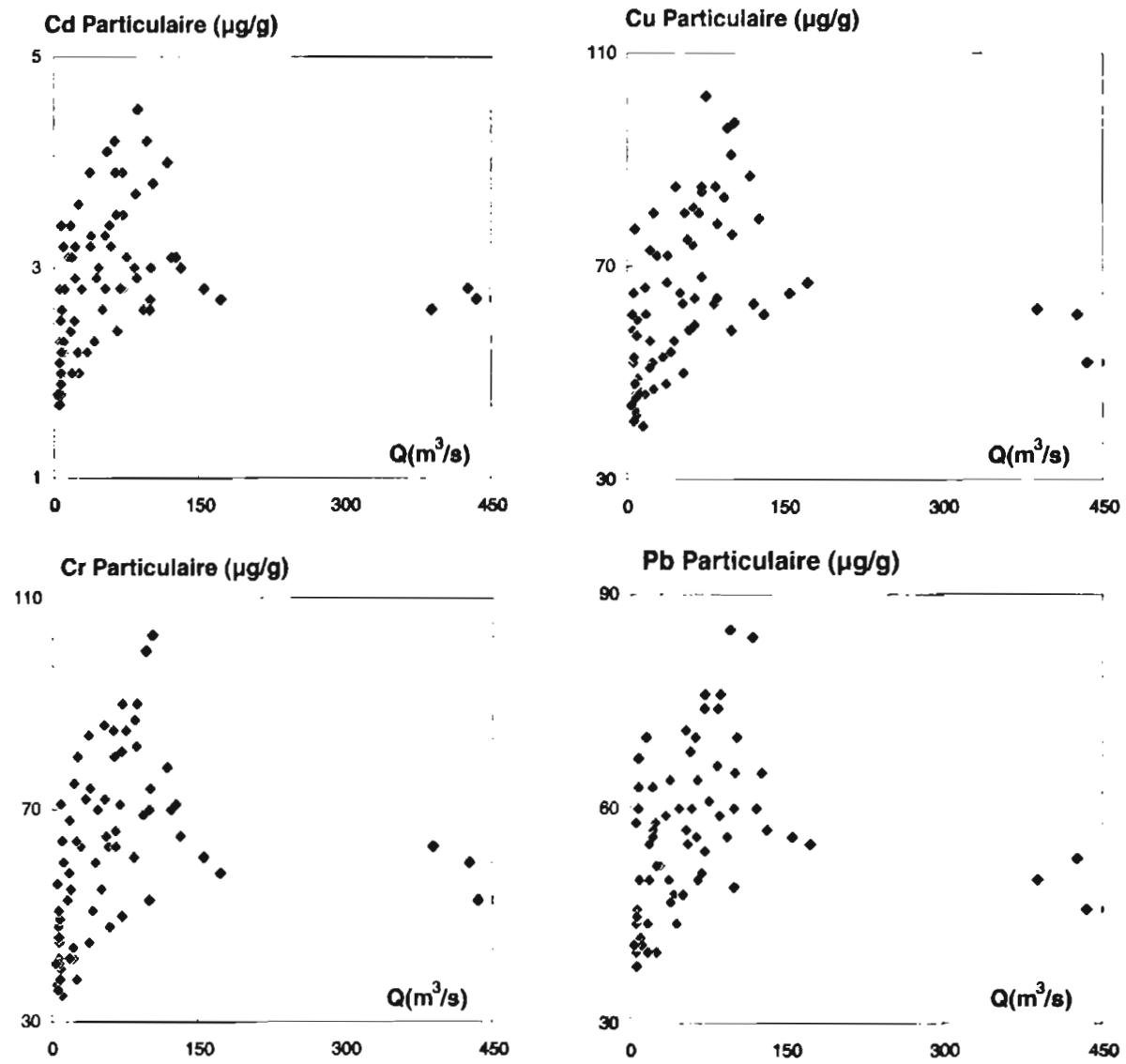

Zn Partlculaire $(\mu g / g)$

Ni Particulaire $(\mu g / g)$ 300

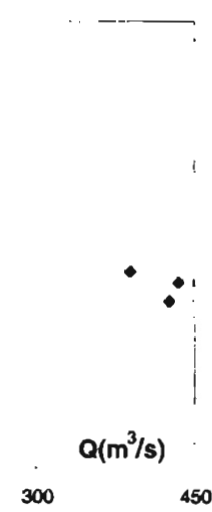

80
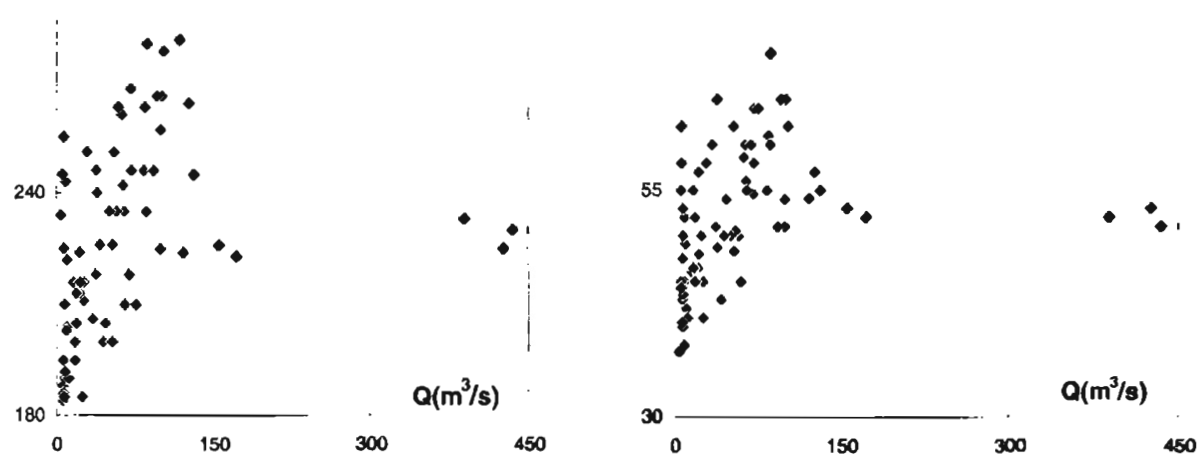

Fig. 5. - Variations des teneurs en métaux traces particulaires dans les eaux de l'Orne en fonction du débit.

Fig. 5. - Variations in particulate trace metal concentrations as a function of Orne flow. 

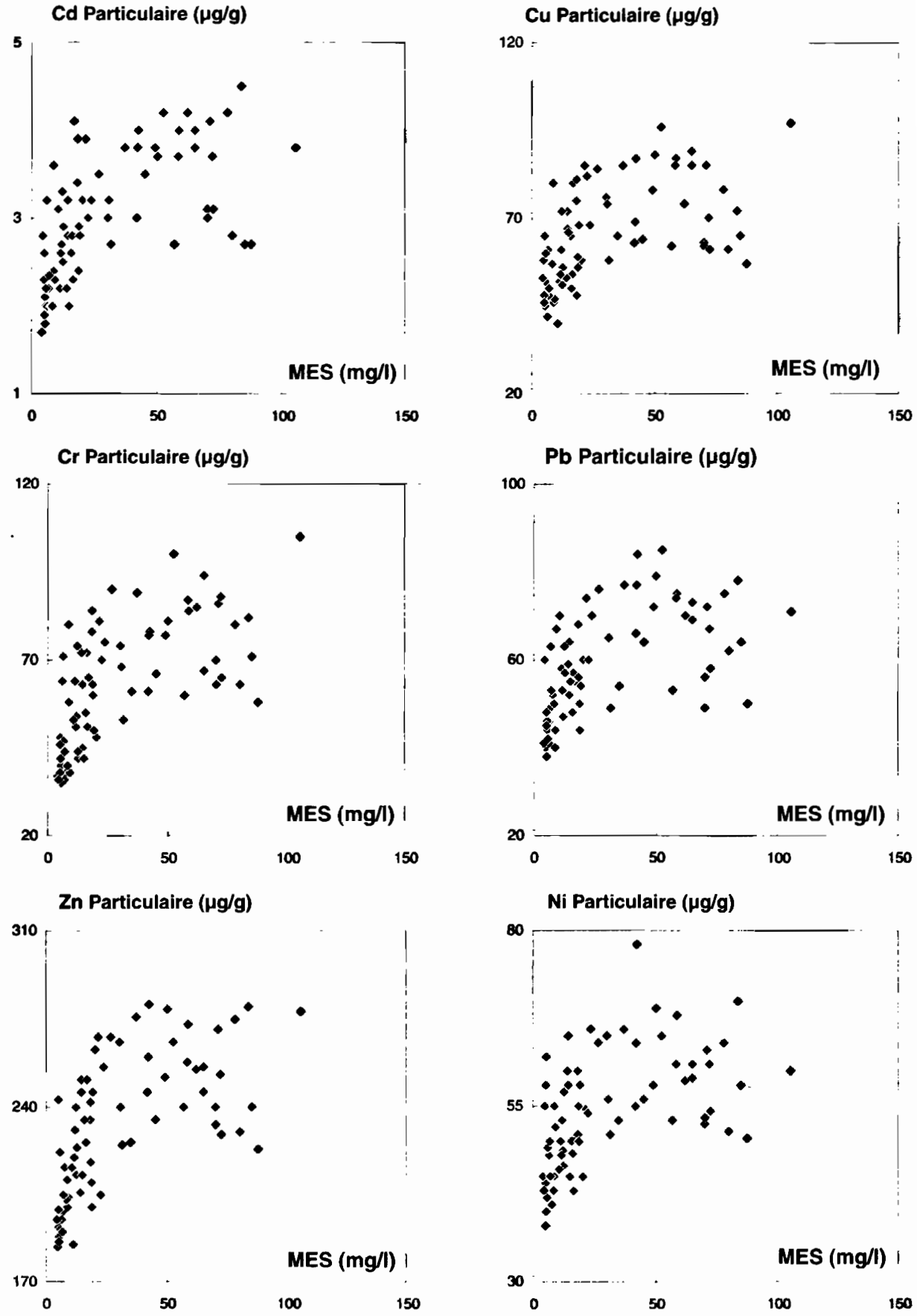

Fig. 6. - Variations des concentrations des métaux en traces avec les MES.

Fig. 6. - Variations in particulate trace metal concentrations as a function of SM in the Orne. 

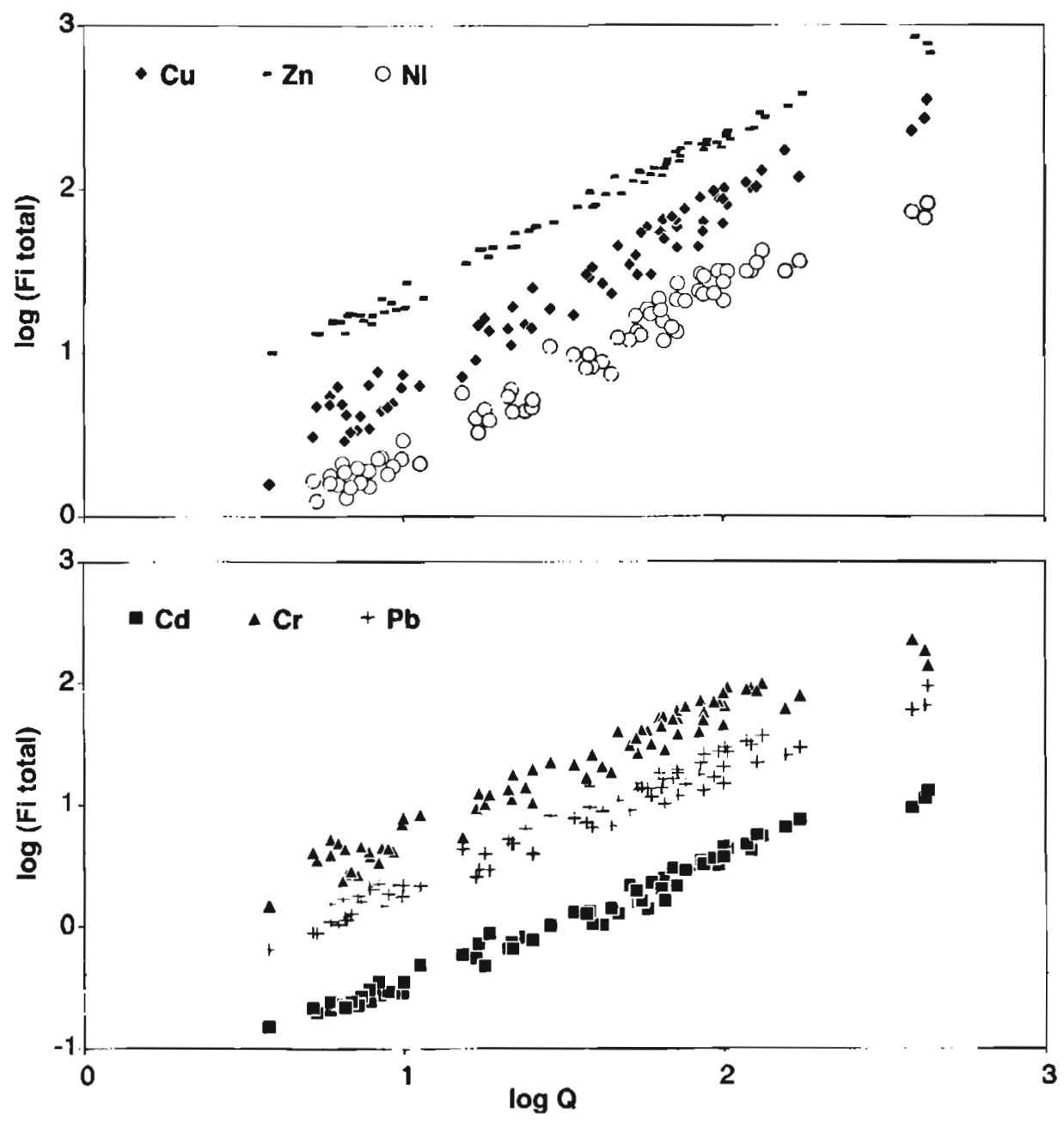

Fig. 7. - Variations des flux instantanés (Fi) des métaux traces dans les eaux de l'Orne. Fig. 7. - Instantaneous total contaminant flux (Fi total) vs river discharge $Q$ ( in log scale)

MES entraînée par la poussée algale estivale. Cependant, certaines teneurs mesurées en étiage sont élevées. Elles peuvent être expliquées par la complexation des métaux en traces sur la matière organique qui constitue en cette période l'essentiel des MES présentes dans les eaux (Lin et Chen, 1998).

Lors de la grande crue de janvierfévrier 1995, on observe pour tous les éléments une chute importante des concentrations de la fraction dissoute due à l'effet de la dilution. 
Les flux instantanés à May/Orne augmentent nettement avec le débit et ne sont que faiblement affectés par la dilution des concentrations de la fraction dissoute (fig. 7). Les gammes de valeurs dans lesquelles varient les débits et les concentrations des MES sont très importantes par rapport à celle dans laquelle varie les concentrations des métaux, comme le montrent les valeurs des coefficients de variation (respectivement 135 et $98,5 \%$ contre $30 \%$ en moyenne).

\subsection{Partition des métaux traces}

Les variations des concentrations des métaux en traces dans les fleuves sont influencées aussi bien par les débits liquides que par la charge solide. Les niveaux apparents des éléments sont régulés par la distribution entre les phases solides et dissoutes (Zhang et al., 1994). Cette répartition est souvent représentée par le coefficient de distribution $\mathrm{K}_{\mathrm{d}}$ donné par la relation :

$$
\mathrm{K}_{\mathrm{f}}=\left(\mathrm{C}_{\mathrm{p}} / \mathrm{C}_{\mathrm{f}}\right)
$$

$C_{p}$ et $C_{f}$ étant les concentrations des formes particulaires et dissoutes exprimées en ( $\mathrm{ng} / \mathrm{Kg}$ de MES) et en (ng.Kg ' d'eau).

Les valeurs moyennes de $K_{d}$ sont comparées dans le tableau VII à celles d'autres fleuves.

Elles varient en moyenne entre $10^{48}$ pour le $\mathrm{Cr}$ et $10^{5.4}$ pour le $\mathrm{Pb}$ à May/Orne. Ces valeurs sont très semblables à celles des autres fleuves. Les valeurs de $K_{d}$ varient généralement dans les mêmes ordres de grandeur, malgré les grandes différences qui peuvent exister entre les bassins versants au niveau des superficies, des débits liquides et solides, de la composition des MES, des processus d'érosion et d'altération et de l'activité anthropique.

Tableau VII. - Coefficients de partition dans l'Orne (May/Orne) et dans d'autres fleuves.

Table VII. - Comparison of $\mathrm{Kd}(\log \mathrm{Kd})$ for trace metals in Orne and some world rivers.

\begin{tabular}{|c|c|c|c|c|c|c|c|}
\hline $\log K d$ & $\mathrm{Cd}$ & $\mathrm{Cu}$ & $\mathrm{Cr}$ & $\mathrm{Pb}$ & $\mathrm{Zn}$ & $\mathrm{Ni}$ & références \\
\hline Orne & 5,0 & 4,9 & 4,8 & 5,4 & 5,0 & 5,1 & cette étude (1993-1995) \\
\hline Seine & 5,3 & 5,0 & - & 5,7 & 4,8 & - & Cossa et al., 1994 \\
\hline Mississippi & 4,6 & 4,2 & - & 5,5 & - & 4,4 & Trefry et al., 1986 \\
\hline Huanghe & 5,5 & 4,2 & - & 6,0 & 5,4 & 4,9 & Zhang et al., 1994 \\
\hline Nile & - & 5,2 & - & 5,2 & - & - & Abdel-Moati, 1990 \\
\hline moyenne mondiale & 4,7 & 4,82 & 5 & 6 & 6,92 & 5,25 & Martin et Whitfield, 1983 \\
\hline
\end{tabular}

Fig. 8. - Variations temporelles du coefficient de partition Kd dans l'Orne (1993-1995).

Fig. 8. - Temporal variations in the partitioning coefficient Kd in the Orne River (1993-1995). 

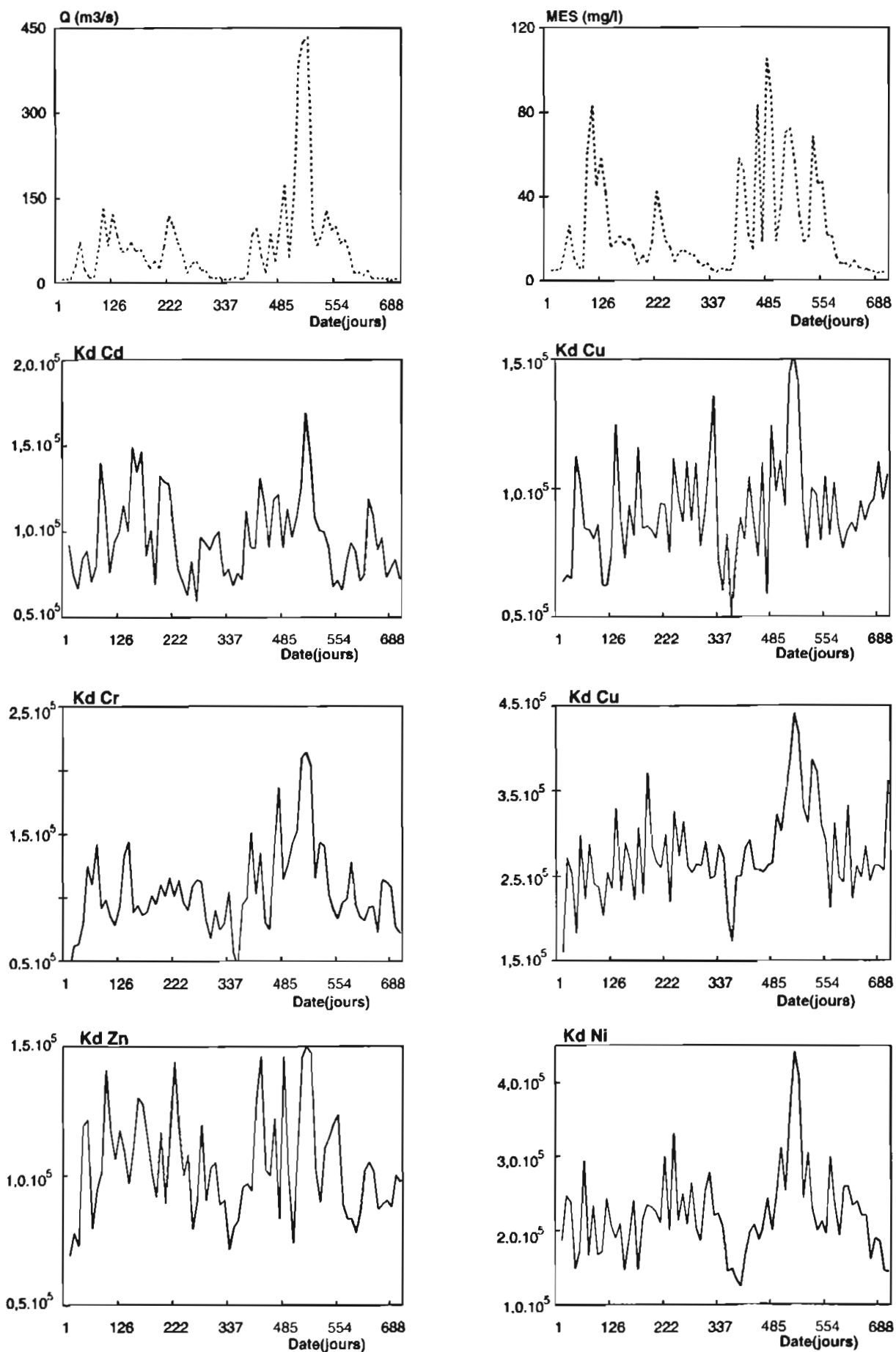
Ces valeurs élevées montrent la grande capacité des particules à piéger les métaux traces.

Les variations saisonnières du coefficient de partition montrent des augmentations systématiques au moment des crues, c'est à dire lors des périodes où la charge en matière en suspension est forte (fig. 8). Ce phénomène traduit la diminution plus forte des concentrations des espèces dissoutes comparativement à celles des espèces particulaires lors des crues. La phase particulaire semble réagir plus lentement que la phase dissoute à la dilution lors des crues.
A partir de l'équation (1), on peut exprimer en fonction de $\mathrm{K}_{a}$ la proportion de la concentration totale $\left(C_{10}\right)$ d'un élément trace transportée sous forme dissoute.

La concentration totale $C_{\text {tot }}$ en ng.kg ', s'écrit :

$$
C_{101}=C_{1}+\text { MES. } C_{p}=C_{d}\left(1+\text { MES } . K_{d}\right)
$$

et on en déduit

$$
\frac{C_{d}}{C_{t o t}}=\frac{1}{1+M E S}{ }^{*} K_{d}^{-}
$$

MES, concentration exprimée en $\mathrm{mg} \cdot \mathrm{I}^{-1}$.

La représentation graphique de ce rapport exprimé en \%, en fonction

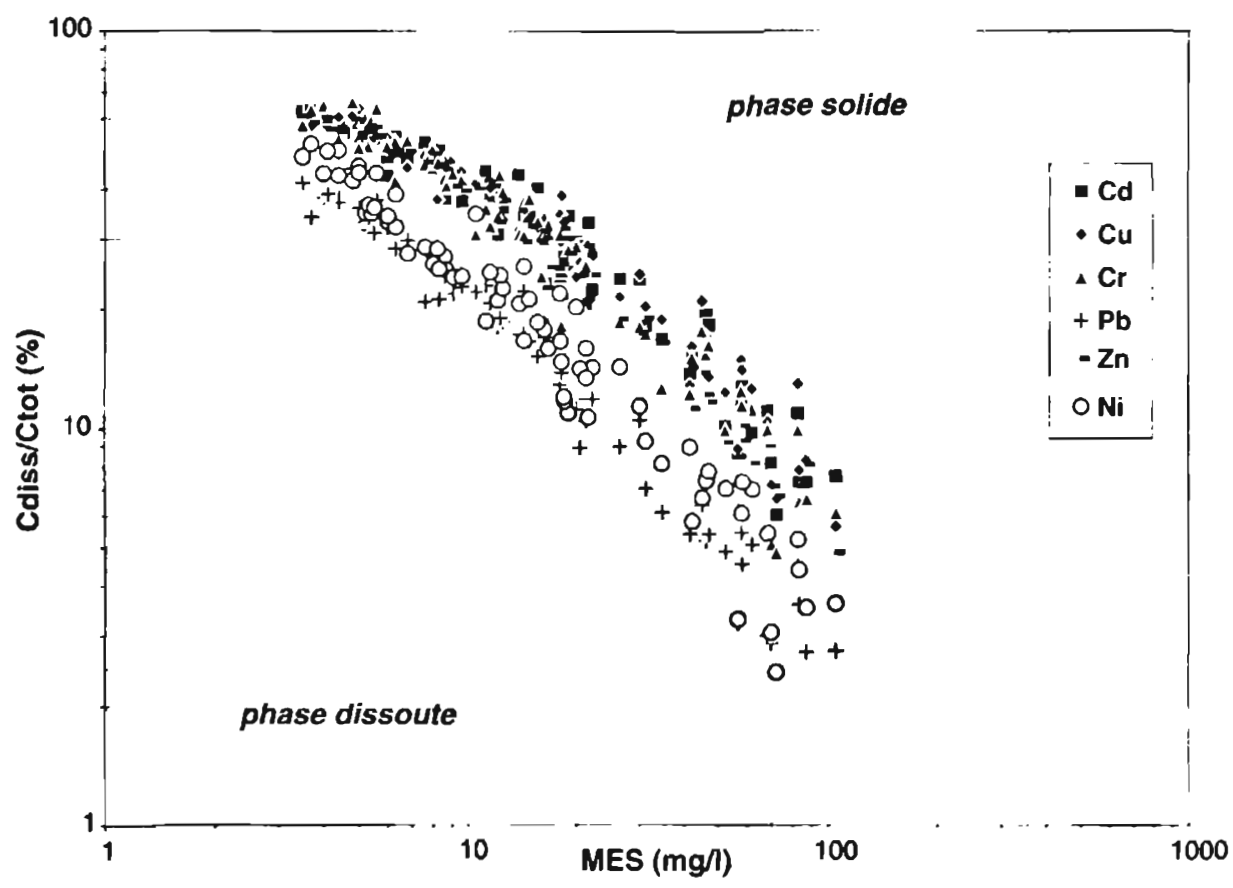

Fig. 9. - Variations du pourcentage des métaux traces dissous avec les MES dans l'Orne. Fig. 9. - Variations in the percentage of dissolved trace metals v.s. suspended sediment concentrations in the Orne river (1993-1995). 
des MES (fig. 9), montre que le pourcentage du métal total transporté sous forme dissoute diminue régulièrement avec le taux des MES. De plus, si on considère une concentration moyenne des MES de $50 \mathrm{mg} . \mathrm{I}^{-1}$, $28 \%$ du métal trace total est lié à la phase dissoute pour une valeur de $\mathrm{K}_{\mathrm{d}}$ de l'ordre de $10^{4,7}$, mais seulement $6 \%$ lui sont liés si $K_{d}$ est de l'ordre de $10^{5.5}$.

Cette décroissance rapide des concentrations des métaux traces dissous, montre que l'augmentation des teneurs en MES des eaux entraîne par adsorption, coprécipitation et/ou autres réactions chimiques, une réduction de l'amplitude des variations des concentrations des métaux traces dissous et de la biodisponibilité. La capacité des particules à réduire les variations des teneurs des éléments dans la phase dissoute dépend principalement des interactions de ces éléments avec la phase solide et des concentrations des sédiments en suspension dans les rivières.

\subsection{Influence de certains paramètres sur le coefficient de partition}

Le traitement statistique des données montre que les variations des teneurs en MES, de la température de l'eau et du $\mathrm{pH}$ ont une influence sur le coefficient de partition $K_{d}$. Nous avons établi à partir de méthodes statistiques utilisant des régressions, des modèles linéaires liant ce coefficient à ces paramètres. Les coefficients de corrélation des relations étalies entre $\log K_{d}$ et ces trois facteurs sont compris entre 0,3 et 0,6 $(n=102)$. Ils montrent que ces facteurs jouent un rôle plus ou moins important dans la partition des métaux traces entre les phases solide et dissoute. La MES est le facteur qui présente la meilleure corrélation avec le $\mathrm{K}_{\mathrm{d}}$. Les conditions favorisant l'association des métaux en trace à la phase solide sont constituées par des températures basses, des $\mathrm{pH}$ élevés et des concentration en MES fortes.

Tableau VIII. - Valeurs des coefficients normalisés $\left(\log K_{\imath}\right)$ de la régression linéaire multiparmétrique établie pour l'Orne en 1993-1995.

Table VIII. - Multiparameter linear regression analysis of partitioning coefficients $\left(\log K_{f}\right)$ in the Orne.

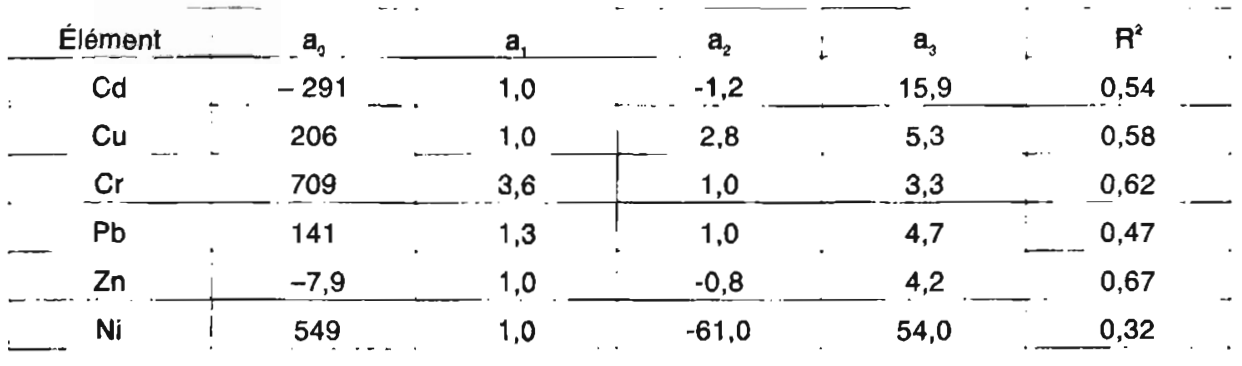


On a, à l'aide d'une analyse des régressions linéaires multiparamétriques, exprimé $\mathrm{K}_{t}$ en fonction de ces trois paramètres et mis ainsi en évidence le poids de chacun de ces 3 facteurs dans les variations du coefficient de partition. Le modèle établi s'écrit sous la forme suivante:

$$
\log \mathrm{K}_{\mathrm{d}}=
$$

$$
\mathrm{a}_{0}+\mathrm{a}_{1} \mathrm{pH}+\mathrm{a}_{2} \mathrm{~T}\left({ }^{\circ} \mathrm{C}\right)+\mathrm{a}_{3} \text { MES }
$$

Les résultats de l'analyse ainsi que les coefficients de corrélation correspondants sont donnés dans le tableau VIII. Ces résultats montrent que la concentration en MES est, de ces facteurs, celui qui a le plus de poids dans les variations qui affectent le coefficient de partition des métaux traces dans l'Orne. On a vu précédemment que les teneurs en métaux traces particulaires sont liées fortement aux concentrations des MES, ainsi qu'à leur nature.

\subsection{Bilan des transferts}

Les estimations des tonnages des deux formes des métaux en trace apportés par l'Orne à l'entrée de son estuaire sont données dans le tableau IX.

Les flux annuels des métaux en trace (dissous et particulaires) dans l'Orne ne représentent qu'une infime partie des flux dissous et particulaires

Tableau IX. - Flux annuels dissous et particulaires des métaux dans l'Orne à May/Orne durant les cycles hydrologiques 1993-1994 et 1944-1995.

Table IX. - Annual fluxes of the dissolved and particulate metals in the Orne at May/Orne for the hy-

\begin{tabular}{|c|c|c|c|c|c|c|c|}
\hline \multicolumn{2}{|c|}{ Flux (Kg/an) } & Cd & $\mathrm{Cu}$ & $\mathrm{Cr}$ & $\mathrm{Pb}$ & $\mathrm{Zn}$ & $\mathrm{Ni}$ \\
\hline \multirow[t]{2}{*}{ Td } & 1993-1994 & 18.4 & 367 & 345 & 252 & 1920 & 263 \\
\hline & $1994-1995$ & 20.4 & 447 & 369 & 270 & 2230 & 280 \\
\hline \multirow[t]{2}{*}{$T p$} & 1993-1994 & 91 & 2140 & 1930 & 1640 & 7340 & 1550 \\
\hline & 1994-1995 & 161 & 3320 & 2840 & 2920 & 12100 & 2410 \\
\hline$T p /(T p+T d)$ & 1993-1994 । & 83 & 85 & 85 & 87 & 77 & 86 \\
\hline$(\%)$ & $1994-1995$ & 89 & 88 & 89 & 92 & 84 & 90 \\
\hline
\end{tabular}
drological cycles 1993-1994 and 1994-1995.

Tableau X. - Flux spécifiques des métaux en traces particulaires ( $/ / \mathrm{km} 2 / a n)$ dans l'Orne, la Seine el l'Adour.

Table X. - Specific particulate fluxes in the Orne, the Seine and the Adour rivers.

\begin{tabular}{lccccccl} 
& $\mathrm{Cd}$ & $\mathrm{Cu}$ & $\mathrm{Cr}$ & $\mathrm{Pb}$ & $\mathrm{Zn}$ & $\mathrm{Ni}$ & \multicolumn{1}{c}{ références } \\
Orne & 0,045 & 0,97 & 0,85 & 0,81 & 3,47 & 0.71 & cette étude (1193-95) \\
Adour & - & 1,10 & 0,88 & 1,10 & 3,00 & 0,50 & Snoussi, 1988 \\
Seine (90-91) & 0,042 & 1,45 & - & 1,55 & 5,82 & - & Cossa et al., 1994 \\
Moselle & - & 2,48 & 3,35 & - & 12.10 & 1,89 & Kattan, 1989
\end{tabular}


annuels totaux minéraux (majeurs, mineurs et traces) entrant dans l'estuaire.

Ainsi, le tonnage annuel des métaux en traces dissous ne représentent que $14.10^{-4} \%$ des transports annuels de matières en solution (243.10 $10^{3}$ /an; Nasseh, 1997), et les tonnages des métaux en traces particulaires ne représentent que $11.10^{-2} \%$ des transports annuels de matières particulaires $\left(17.10^{3} \mathrm{t} / \mathrm{an}\right.$; Nasseh, 1997).

Les flux spécifiques dissous annuels montrent, notamment pour le cadmium, des valeurs supérieures à celles de la Seine, et des valeurs comparables à celles de l'Adour. Les flux spécifiques particulaires, les tonnages sont légèrement inférieurs à ceux de la Seine (sauf pour Cd) et très faibles par rapport à ceux de la Moselle (tab. X).

L'exportation des éléments s'effectue essentiellement, comme c'est généralement le cas, sous forme particulaire ( $86 \%$ en moyenne). Ce pourcentage augmente notablement durant le cycle 1994-1995 du fait particulièrement de l'effet de dilution de la crue exceptionnelle de janvier
1995 sur les concentrations dissoutes. Les flux totaux exportés durant le cycle 1994-1995 (27,3 tonnes), qui fut plus humide, sont supérieurs de près de $24 \%$ à ceux exportés au cours du cycle 1993-1994 (17,8 t). Les contributions moyennes relatives des transports dissous $T_{d}$ et des transports particulaires $T_{p}$ donnent un rapport $T_{d} / T_{p}$ faible de l'ordre de 0,21.

Cette partition des exportations entre les phases dissoute et particulaire dans le bassin de l'Orne est comparable à celle observée dans la majorité des fleuves mondiaux.

Les contributions des différents épisodes de crue au tonnage total des métaux en traces évacués par l'Orne témoignent de l'importance et de l'efficacité de ces épisodes dans des intervalles de temps très courts (tab. XI). A titre d'exemple, la crue de janvier 1994 a exporté, à elle seule, $48 \%$ du tonnage total exporté au cours du cycle 1993-1994. Celle de février 1995 a exporté près de $53 \%$ des apports en métaux en traces du cycle 1994-1995. Les tonnages exportés par l'ensemble des crues représentent 63 et $67 \%$ des flux des métaux en traces en 1993-1994 et 1994-1995.

Tableau XI. - Contributions et tonnages des métaux traces exportés au cours des crues (tonnes).

Table XI. - Fluxes of trace metals exported during floods (in tonnes).

crues cycle 1993-1994 octobre janvier avril

tonnages transportés

$\%$ du tonnage total

$\begin{array}{ccc}0.49 & 10.6 & 1.92 \\ 2.4 & 51 & 9.3\end{array}$
novembre décembre : janvier février

$\begin{array}{cccc}1.38 & 0.57 & -1.63 \\
5.4 & 2.2\end{array}-6.3 \quad$\begin{tabular}{c}
13.7 \\
\hline
\end{tabular}




\section{CONCLUSION}

L'étude des transports des métaux en traces menée dans le bassin de l'Orne a été effectuée à partir des données recueillies au cours de deux cycles hydrologiques. Les prélèvements ainsi que les analyses ont été effectuées dans des conditions ultrapropres afin d'éliminer toute source de contamination.

Les deux cycles durant lesquels s'est déroulée cette étude sont très humides. Les débits moyens annuels des deux cycles sont supérieurs de 60 et $90 \%$ au débit moyen interannuel $\left(20,5 \mathrm{~m}^{3} / \mathrm{s}\right)$ calculé sur la période 1984-1993. L'Orne a connu en janvier-février 1995 une crue centennale qui a duré plusieurs jours et atteint $452 \mathrm{~m}^{3} / \mathrm{s}$ soit plus de 20 fois le débit interannuel moyen.

Les niveaux observés dans le bassin ont permis d'identifier une contamination relativement importante en cadmium d'origine anthropique qui est probablement en relation avec l'utilisation massive des engrais phosphatés en agriculture.

Cette contamination est confirmée aussi bien par les calculs du facteur d'enrichissement que du taux de dénudation. Par contre, les concentrations de la plupart des autres métaux sont comparables aux niveaux observés dans les substrats du bassin versant, ce qui peut témoigner d'une origine essentiellement naturelle pour ces métaux. Le comportement de la phase particulaire, toujours dominante, est très dépendant des va- riations du débit et de la charge solide. La phase dissoute est plus affectée par la dilution que la phase particulaire, et notamment lors des crues.

Les valeurs du coefficient de partition entre la phase dissoute et la phase particulaire sont semblables à ceux observés sur les fleuves mondiaux. Leurs variations temporelles suivent globalement les variations des débits et de la charge solide. La concentration en MES dans les eaux est le facteur le plus influent sur cette partition.

Les exportations des métaux en trace s'effectuent essentiellement sous forme particulaire $(86 \%$ en moyenne). Ce pourcentage augmente notablement durant les crues du fait de l'effet plus important de la dilution sur la phase dissoute.

\section{RÉFÉRENCES}

Abdel-Moati A.R., 1990. Behaviours and fluxes of copper and lead in the Nile river estuary. Estuarine, Coastal Shelf Sci., $30:$ 153-165.

Cossa D., Meybeck M., Idlafkih Z. et Bombled B., 1994. Étude pilote des apports en contaminants par la Seine. Rapport IFREMER-ME-AESN, $89 \mathrm{p}$ et annexes.

Edwards A.M.C., 1973. The variation of dissolved constituents with discharge in some Norfolk rivers. J. Of Hydrology, $18: 219-224$.

Elbaz-Poulichet F. et Martin J.M., 1987. Dissolved Cd behaviour in some selected french and chinese estuaries. Consequences on $\mathrm{Cd}$ supply to the Ocean. Mar. Chem., 22 : 125-136. 
Guieu C., Huang W.W., Martin J.M. et yong y.y., 1996. Outflow of trace metals into the Laptev Sea by the Lena river. Mar. Chem., 53, 3-4: 255-267.

Idlafkih Z., Cossa D, et Meybeck M., 1995. Comportements des contaminants en trace dissous et particulaires (AS, Cd, Cu, Hg, Pb, $\mathrm{Zn}$ ) dans la Seine. Hydroécol. Appl., 7, 1-2 : 127150.

Idlafkih Z., Meybeck M., Chiffoleau J.F., Cossa D. et Ficht A., 1997. Comportements des contaminants en trace dissous et particulaires (Al, Fe, Mn, Cd, $\mathrm{Cu}, \mathrm{Hg}, \mathrm{Pb}$ et $\mathrm{Zn}$ ) dans la Seine a Poses en période de hautes eaux (19901995). Proc. Rabat Symp., IAHS Publ., $243: 45-58$.

Kattan Z., 1989. Géochimie et hydrologie des eaux fluviales des bassins de la Moselle et de la Mossig. Transport dissous et particulaires. Cycles biogéochimiques des éléments. Thése Doc., Université Louis Pasteur, Strasbourg, $218 \mathrm{pp}$.

Lin J. and Chen S.Y., 1998. The relationship between adsorption of heavy metal and organic matter in river sediments. Environ. International, 23, $3: 345-352$.

Martin J.M. et Whitfield M., 1983. The significance of the river input of chemical elements to the Ocean. In : Trace metals in sea water. New York, Plenum Press : 265-296.

Meade R.H. and Parker R.S., 1984. Sediment in the rivers of the United States. Nat. Wat. Summ. 1984. U.S. Geol. Surv. Wat. Supp. Paper, 2275 : 49-69.

Meybeck M., 1984. Les fleuves et le cycle géochimique des éléments. Thèse Doc. ès Sciences, Univ. de Paris VI, 554 pp.

Meybeck M., 1998. Man and river interface; multiple impacts on water and particulates chemistry illustrated in the Seine river basin. Hydrobiologia, 373$374: 1-20$.

Mueller G. and Furrer R., 1998. Pollution of the River Elbe; past, present and fu- ture. Water Quality International, IAWPRC, 15-18.

Nasseh A., 1997. Flux et variabilité spatio-temporelle des transports dans le bassin de l'Orne. Thèse Doc., Université Libre de Bruxelles, 207 pp.

Nasseh A., Texier H., Lacroix M. et Verague J., 1996. Variabilité saisonnière des flux géochimiques dans un petit bassin versant tempéré, exemple de l'Orne (Calvados-France). Geomorphologie, Relief, Processus, Environnement, $3: 57-92$.

Nasseh A., Texier H., Lacroix M. et Ouddane B., 1999. Transport de la charge solide dans le bassin versant de l'Orne. Composition, variabilité et flux. Hydroécologie Appliquée, 11, 1-2: 103-125.

Ouddane B., 1990. Comportement des éléments majeurs et mineurs dans un milieu soumis à des gradients physicochimiques marqués: cas de l'estuaire de la Seine. Thèse Doc., U.S.T.L., 227 pp.

Shankar R. et Manjunatha B.R., 1994. Elemental composition and particulate metal fluxes from Netravati and Gupur rivers to the Coastal Arabian Sea. J. Geol. Soc. India, 43 : 255-265.

Snoussi M., 1988. Nature, estimation et comparaison des flux de matières issus des bassins versants de l'Adour (France), du Sebou, de l'Oum-er-Rbia et du Souss (Maroc). Mémoire de I'Institut de Géologie du Bassin d'Aquitaine, 22, $459 \mathrm{pp}$.

Taleb A., 1997. Pollutions diffuse ou localisée des eaux de la Senne en amont de Tubize (Belgique) par les nutriments. Thèse Doc., Univ. Libre de Bruxelles, $239 \mathrm{pp}$.

Trefry J.M., Nelson T.A., Trocine R.P., Metz S. et Vetter T.W., 1986. Trace metal fluxes through the mississipi river delta system. Rapp. P.-V. Reun. Cons. Int. Explor. Mer, 186 : 277-288.

Van Der Weijden C. H. et Middelburg G.J.J., 1989. Hydrogeochemistry of 
the river Rhine, long term and seasonal variability, elemental budgets, base levels and pollution. Wat. Res., 23, $10: 1247-1266$.

Zhang J. et Huang W.W., 1993. Dissolved trace metals in the Huanghe : the most turbid large river in the world. Water Res., $27: 1$-8.

Zhang J., Huang W.W. et Wang J.H., 1994. Trace-metal chemistry of the Huanghe, China- Examination of the data from in situ measurments and la- boratory approach. Chemical Geology, 114 : 83-94.

Zhang J., 1995. Geochemistry of trace metals from chinese river/estuary systems: an overview. Estuar. Coast. Shelf Sci., 41, $6: 631-658$.

Zhang J., Huang W.W., Letolle R. et Jusserand C., 1995. Major element chemistry of the Huanghe (Yellow river), China. Weathering processes and chemical fluxes. J. Hydrol. Amst., 168, 1-4:173-203. 